This item was submitted to Loughborough's Research Repository by the author.

Items in Figshare are protected by copyright, with all rights reserved, unless otherwise indicated.

\title{
Learning object-centric complementary features for zero-shot learning
}

PLEASE CITE THE PUBLISHED VERSION

https://doi.org/10.1016/j.image.2020.115974

PUBLISHER

Elsevier

VERSION

AM (Accepted Manuscript)

PUBLISHER STATEMENT

This paper was accepted for publication in the journal Signal Processing: Image Communication and the definitive published version is available at https://doi.org/10.1016/j.image.2020.115974.

\section{LICENCE}

CC BY-NC-ND 4.0

\section{REPOSITORY RECORD}

Liu, Jie, Kechen Song, Yu He, Hongwen Dong, Yunhui Yan, and Qinggang Meng. 2020. “Learning Objectcentric Complementary Features for Zero-shot Learning". Loughborough University.

https://hdl.handle.net/2134/12962837.v1. 


\title{
Learning Object-centric Complementary Features for Zero-shot Learning
}

Jie Liu ${ }^{1,2}$, Kechen Song ${ }^{1,2^{*}}$, Yu He ${ }^{1,2}$, Hongwen Dong ${ }^{1,2}$, Yunhui Yan ${ }^{1,2^{*}}$, Qinggang Meng ${ }^{3}$

1. School of Mechanical Engineering \& Automation, Northeastern University, Shenyang, Liaoning, China

2. Energy Saving Metallurgical Equipment and Intelligent Detection Engineering Technology Research Center of Liaoning Province, Shenyang, Liaoning, China

3. Department of Computer Science, Loughborough University, Loughborough, LE11 3TU, U.K.

\begin{abstract}
:
Zero-shot learning (ZSL) aims to recognize new objects that have never seen before by associating categories with their semantic knowledge. Existing works mainly focus on learning better visual-semantic mapping to align the visual and semantic space, while the effectiveness of learning discriminative visual features is neglected. In this paper, we propose an objectcentric complementary features (OCF) learning model to take full advantage of visual information of objects with the guidance of semantic knowledge. This model can automatically discover the object region and obtain fine-scale samples without any human annotation. Then, the attention mechanism is used in our model to capture long-range visual features corresponding to semantic knowledge like 'four legs' and subtle visual differences between similar categories. Finally, we train our model with the guidance of semantic knowledge in an end-to-end manner. Our method is evaluated on three widely used ZSL datasets, CUB, AwA2, and FLO, and the experiment results demonstrate the efficacy of the object-centric complementary features, and our proposed method outperforms the state-of-the-art methods.
\end{abstract}

Keywords: Zero-shot learning; Object-centric complementary features; Attention mechanism; Image recognition; Salient object detector.

\footnotetext{
*Corresponding author. Email: songkc@ me.neu.edu.cn (Kechen Song), yanyh@ mail.neu.edu.cn (Yunhui Yan). 


\section{Introduction}

Recently, deep learning has achieved great success in object recognition tasks [6,12,29, 34, $36,52]$, and in some cases, can even be comparable to human recognition capacity. However, deep learning approaches require large-scale labeled samples to achieve satisfactory performance. Data collection and annotation are usually laborious and even require specialized domain knowledge, especially for rare and fine-grained object categories. Moreover, it is a challenge for deep learning methods to recognize new classes after the training stage. By contrast, human can recognize new objects only from a description in terms of concepts in some semantic vocabulary [1]. For example, it is easy for a child to recognize a mermaid by telling him that mermaid is a creature with human body and fishtail, even never seeing a mermaid before. Inspired by the ability of human to recognize new objects, zero-shot learning (ZSL) aims to recognize new categories that never appear in the training set. In recent years, ZSL has attracted extensive attention in the object recognition task $[3,4,10,11,23,26,32,40]$.

In the ZSL task, the labeled training classes and the unlabeled test classes are entirely disjoint. Based on the visibility of the instance labels, the training classes and the test classes are usually referred to as seen and unseen classes, respectively [33]. To achieve ZSL, auxiliary information (i.e., semantic knowledge) is required to transfer knowledge from seen classes to unseen classes. Some of the most popular semantic knowledge in ZSL are user-defined attributes [2], word vector representations of class names [46], and textual descriptions [45]. Based on semantic knowledge, inter-class relationships can be established. Therefore, attributes such as 'four-legs,' 'furry,' and 'horns' are shared among all classes in the dataset AwA2, which 
can be used to transfer from seen classes 'deer' and 'buffalo' to predict unseen class 'antelope.'

Most existing ZSL methods, whether deep feature-based [13, 14, 16], or end-to-end trainable convolutional neural network $(\mathrm{CNN})$ based approaches $[7,22,26]$, focus on learning better mapping between the global image (or feature) and the corresponding semantic vector. However, the discrimination and generalization of visual features are neglected by those works. As the whole image contains the global information about the object, the global image (or feature) is beneficial to ZSL generalization, i.e., the model trained on seen classes can well recognize unseen classes. In addition, compared with the global feature, the local feature is more discriminative when recognizing objects that have similar visual appearance (e.g., antelope, and deer in Fig. 1), because the local feature can capture subtle visual differences between the similar objects. Therefore, to improve ZSL performance, both the discrimination and generalization of visual features should be taken into consideration.

Specifically, the discrimination and generalization abilities of existing ZSL methods are highly influenced by two crucial factors: (1) Undiscriminating visual features. Existing input images contain many useless backgrounds that can be regarded as interference information, which makes the learning system unaware of which part of the image should be focused on or weakens the discrimination of visual features. As shown in Fig. 1, many parts of the 'antelope' and 'deer' in images are occupied by backgrounds that are useless for the recognition of the corresponding object, which affects the performance of ZSL. (2) The global and local features of objects contain complementary information on different scales. Learning global features independently is easy to ignore some subtle discriminative features, which leads to the poor 
performance of the learned model in recognizing objects with similar visual appearance, such as 'antelope' and 'deer'. Additionally, we can improve the model accuracy on seen classes by learning local discriminative features, but the model generalization on unseen classes will be weakened. Therefore, as shown in Fig. 1, it is easy for a model learned on seen class 'zebra' to recognize zebra merely by the local visual features, but it does not work on unseen class 'tiger', because of the information loss of other features (i.e., global features). As a result, effectively reducing interference information of input image and leveraging complementary information is meaningful and challenging for the improvement of ZSL performance.

In this paper, we propose an end-to-end trainable model which learns object-centric complementary features for ZSL in visual space. We first enable our model to automatically discover the object-centric region and obtain fine-scale images to reduce undiscriminating visual information. Then attention mechanism is used to capture global and local features with the guidance of semantic knowledge, and we fuse them to obtain complementary visual features. Finally, we train our model with the guidance of semantic knowledge in an end-to-end manner. Our method is evaluated on three widely used ZSL datasets, i.e., CUB, AwA2, and FLO, and the experiment results demonstrate that our proposed method outperforms the state-of-the-art methods by $2 \%, 2.3 \%$, and $3.4 \%$, respectively.

The main contributions of this paper are summarized as follows:

1) An object-centric region refine module based on salient object detection is proposed to reserve object region and reduce interference information of the input image. And this is the first time to introduce salient detection methods to improve ZSL performance. 
2) To capture the complementary information of the global and local features, the global discriminative attention (GDA) module and local discriminative attention (LDA) module are proposed respectively.

3) An end-to-end network for ZSL. The discrimination and generalization of ZSL can be improved by learning object-centric complementary features in an end-to-end manner.

\section{Related works}

\subsection{Conventional ZSL Methods}

As a challenging and attractive research field, ZSL has received extensive attention over the last decade. As summarized in [5], most existing ZSL methods can be divided into three categories, attribute methods [19, 20, 21], embedding methods [22, 23], and hybrid methods [24, 25]. As the pioneering work and classical attribute methods of ZSL, [19] first learns attributes classifiers and then calculates the posterior probabilities of every unseen class for a given test image. [20] and [21] combine visual features and semantic features by bilinear compatibility function, in which the linear mapping matrix is learned by hinge ranking loss. Some other methods adopt embedding methods. [22] proposes a piece-wise linear model by learning multiple linear mappings with the selection of which being a latent variable. [23] proposes a deep ZSL model that can improve the features expression ability by adding nonlinear active function Rectified Linear Unit (ReLU). The hybrid methods are designed to represent images and the semantic classes embedding as a mixture of seen class proportions. [24] introduces composite classifiers of unseen classes by combing classifiers of seen classes linearly. [25] provides a generative framework for ZSL by modeling the conditional distribution 
of each class as a multivariate Gaussian distribution. By expanding the search label space to both seen and unseen classes during testing, ZSL becomes Generalized zero-shot learning (GZSL), which is not the focus of this paper, interested readers can refer to [5].

\subsection{Discriminative ZSL Methods}

In the ZSL task, the final classification performance is directly affected by the discrimination of semantic knowledge and visual features, and there are many works to explore the discrimination of latent semantic knowledge and visual features. [35] propose to explore latent discriminative attributes by training seen class classifiers over the latent attributes. In [47], the discrimination of latent attributes is achieved by minimizing the intra-class distance between the attributes. [44] further improves the performance of ZSL by learning complementary attributes. [43] proposes a latent feature guided attribute attention framework for semantic disambiguation.

More related to our work, there are some end-to-end methods to solve the problem that visual features in ZSL are less discriminative. [23] proposes a deep model that integrates multiple semantic features and maps semantic features to visual feature space for classification. To capture discriminative visual features, a zooming mechanism is proposed in [26]. [11] proposes an unbiased embedding method Quasi-Fully Supervised Learning (QFSL) for ZSL, and it is worth noting that QFSL adopts a transductive manner, which means the model can be trained with the data from both seen and unseen classes during the training stage. By the way, our model is trained in an inductive manner. 


\subsection{Attention Mechanism}

Attention mechanism, widely studied and utilized in recent years, has been successfully applied in many computer vision tasks, such as image classification [6,12], semantic segmentation [27], and visual reasoning [28]. Inspired by the successful application of attention mechanisms in computer vision tasks, some works $[30,31,32,40]$ attempt to introduce attention mechanisms to improve the discrimination of visual features in the few-shot and zero-shot learning task. A multi-attention network is proposed in [32] to solve one-shot learning problem. [40] builds an attention attractor network to regularize the learning of novel classes in few-shot learning task. A stacked semantic-guided attention model is proposed in [30] for fine-grained ZSL, which is capable of discovering discriminative subtle visual differences with the guidance of semantic. In the zero-shot retrieval task, [31] proposed a decoupled metric learning framework to learn discriminative visual attributes.

Compared with the aforementioned attention-based methods, our method differs in three aspects : (1) We learn local discriminative details by convolutional responses. (2) the dependency of similar parts of object is established by the global discriminative attention module, visual features corresponding to more global semantic knowledge (e. g. 'four-legged', 'two-wing') can be captured effectively. (3) global features and local details are learned jointly with the model training process to ensure better discrimination and generalization.

\section{Methodology}

The framework of the proposed method (OCF) is shown in Fig. 2, which consists of two main parts: the object-centric region refine network and the complementary features learning 
network (CFNet). The object-centric region refine network is used to predict the object-centric region and then obtain fine-scale images. The complementary features learning network includes two vital modules, the global discriminative attention (GDA) module, and the local discriminative attention (LDA) module. The GDA modules are leveraged to discover more discriminative global visual features, while the LDA module is proposed to capture the subtle visual difference of objects. To achieve ZSL, semantic space embedding is adopted in our method, which is the most popular strategy in the end-to-end ZSL framework.

\subsection{Notations}

We begin by introducing some symbols and defining the problem of our interest. In the ZSL task, $\mathcal{X}, \mathcal{Y}$, and $\mathcal{K}$ represent visual space, label space, and semantic space. Suppose that the training set is defined as $\mathcal{S}=\left\{\left(x_{i}^{s}, y_{i}^{s}, \boldsymbol{k}_{i}^{s}\right) \mid x_{i}^{s} \in \mathcal{X}^{\mathcal{S}}, y_{i}^{s} \in \mathcal{Y}^{\mathcal{S}}, \boldsymbol{k}_{i}^{s} \in \mathcal{K}^{\mathcal{S}}\right\}_{i=1}^{N^{s}}$, where $\boldsymbol{x}_{i}^{s}$ denotes the $i$-th training image (totally $N^{s}$ images) of the seen class, $y_{i}^{s}$ is the corresponding class label of image $x_{i}^{s}$, and $\boldsymbol{k}_{i}^{s}$ is the class embedding of class $y_{i}^{s}, e . g$. , attributes. In addition, the test set is defined as $\mathcal{U}=\left\{\left(x_{i}^{u}, y_{i}^{u}, \boldsymbol{k}_{i}^{u}\right) \mid x_{i}^{u} \in \mathcal{X}^{\mathcal{U}}, y_{i}^{u} \in \mathcal{Y}^{\mathcal{U}}, \boldsymbol{k}_{i}^{u} \in \mathcal{K}^{\mathcal{U}}\right\}_{i=1}^{N^{u}}$, where $\boldsymbol{x}_{i}^{u}, y_{i}^{u}$, and $\boldsymbol{k}_{\boldsymbol{i}}^{\boldsymbol{u}}$ denote the $i$-th unseen image, its label, and class embedding, respectively. The seen and unseen classes are disjoint, i.e., $\mathcal{Y}^{\mathcal{S}} \cap \mathcal{Y}^{u}=\varnothing$. At test stage, given an unseen image $x_{i}^{u}$ and the semantic annotation of the unseen classes $\boldsymbol{k}_{i}^{u}$, ZSL aims to predict the corresponding class label $y_{i}^{u^{*}}$ for $x_{i}^{u}$.

\subsection{Object-centric region refine network}

It has been shown that the learning of object regions is beneficial to the object classification task at the image level $[37,38]$. And we speculate that reserving the object-centric regions and reducing useless background is beneficial to ZSL. [26] propose use two stacked 
fully-connected layers to capture object regions for ZSL and achieves good performance, but the object region is not accurate enough. In this paper, motivated by the advantages of salient object detectors (e.g., EGNet [50], BASNet [51], PAGE-Net [53], and SCRN [54]), BASNet is used to predict the object region and then obtain fine-scale image.

As shown in Fig. 2, the coarse-scale image $x_{c}$ containing a large amount of interference information is fed into BASNet, which outputs the binary image $x_{s}$. In $x_{s}$, the object region is reserved and useless background is neglected. Then a search algorithm, pixel by pixel traversal, is used to localize the candidate region of target object, i. e. minimum enclosing rectangle. And the candidate region is defined as a rectangular region. Assume the top-left corner in original images as the origin of a pixel coordinate system, whose m-axis and n-axis are defined from left-to-right and top-to-bottom, respectively. Then the candidate region can be parameterized by four parameters:

$$
\left[m_{t l}, n_{t l}, m_{b r}, n_{b r}\right]=S\left(\phi_{s}\left(x_{c}\right)\right)
$$

where $\boldsymbol{m}_{t l}$ and $\boldsymbol{n}_{t l}$ indicate the $\mathrm{m}$-axis and $\mathrm{n}$-axis coordinates for the top left corner of the candidate region, respectively, $m_{b r}$ and $n_{b r}$ indicate the m-axis and n-axis coordinates for the bottom right corner. $x_{c}$ is a coarse-scale image, i.e., input of salient object detector, $S(\cdot)$ indicates candidate region localization operation and $\phi_{s}(\cdot)$ represents salient object detection operation.

After obtaining the location parameterized by aforementioned four parameters of the candidate region, the candidate region can be obtained by directly cropping from the coarsescale image $x_{c}$. To facilitate the backward-prorogation, we utilize the sigmoid function to 
produce a two-dimension continuous salient mask $\mathrm{M}$, and its elements $M(m, n)$ can be defined as:

$$
\begin{aligned}
& M_{m}=f\left(m-m_{t l}\right)-f\left(m-m_{\mathrm{b} r}\right) \\
& M_{n}=f\left(n-n_{t l}\right)-f\left(n-n_{b r}\right)
\end{aligned}
$$

where $\mathrm{m}$ and $\mathrm{n}$ are the horizontal and vertical coordinates of the pixel coordinate system, respectively. And $f(x)=1 /(1+\exp (-t x)), \mathrm{t}$ is a hyper-parameter and is set to 10 in all the following experiments.

Then the candidate region can be obtained by implementing element-wise multiplication between the coarse-scale image $x_{c}$ and the salient mask M:

$$
x_{f}=x_{c} \otimes M
$$

Finally, to extract effective feature representation from the object-centric region, bilinear interpolation is used to adaptively zoom the cropped region to the size of the original image $x_{c}$. Then the zoomed fine-scale image $x_{f}$ are fed into the CNN backbone to extract discriminative feature representation.

\subsection{Complementary features learning network (CFNet)}

\subsubsection{Global discriminative attention module}

The long-range visual features related to semantic knowledge, such as 'four-legs' and 'long-neck' are important for model to recognize object precisely. And these more global features can be obtained by capturing long-range context information of input images. Many works adopt local discriminative visual features as visual representations, but it is noticeable that learning local features independently usually causes overfitting and poor generalization of unseen classes. Inspired by [39], the GDA module is introduced to capture the long-range 
context relationship between the local features related to semantics. With the help of the GDA module, ZSL model can capture more global visual features, which leads to better ZSL performance. The framework of GDA is illustrated in Fig. 3.

Given the output feature maps of the $\mathrm{CNN}$ backbone $F \in \mathbb{R}^{C \times H \times W}$, we first reshape it into three same feature maps $X \in \mathbb{R}^{C \times N}$, where $N=H \times W$, and then multiply the transpose of $X$ by $X . X^{T} X$ represents the relationship between different position of feature maps. Specifically, $X_{i}$ is the $i$-th feature map and $\left(X^{T} X\right)_{i, j}$ denotes the impact of the $i$-th position on the $j$-th position on the feature map. The more similar feature representations of the two locations lead to their stronger correlation. Then we multiply $X$ by $X^{T} X$ and the result is reshaped to $\mathbb{R}^{C \times H \times W}$. Finally, we multiply it by a scale parameter $\lambda$ and implement an element-wise sum operation with feature maps $F$ to obtain the final output feature maps $G \in \mathbb{R}^{C \times H \times W}$ :

$$
G_{i}=F_{i}+\lambda \sum_{j=1}^{N} X_{j}\left(X^{T} X\right)_{i, j}
$$

where $\lambda$ is initialized as 0 and gradually learns to assign more weight. We can observe from the Eq. (4) that the value of every position in $G$ is the weighted sum of original features $F$ and features of all positions. Therefore, similar features promote each other, then the compact and semantic consistency of visual features corresponding to different semantic will be improved. Visual features that correspond to semantic knowledge like 'four-legs' and 'two-wings' can be captured effectively due to the application of the GDA module. Compared with the CNN backbone, the GDA module can capture more global features of the input images.

\subsubsection{Local discriminative attention module}

Global features can be used to well recognize objects with large appearance differences. 
However, it is difficult to use global features to distinguish objects that share high similarity on visual appearance, as the subtle visual feature differences between them are not well learned, or ZSL networks do not pay enough attention to it. Therefore, we leverage the local features to identify similar objects, because local features can discover the subtle differences of visual features, which correspond to the same semantic concept between different categories. In this paper, the GDA module is used to discover these discriminative details.

Specifically, as illustrated in Fig. 4, we take the output feature map of the GDA module as $G \in \mathbb{R}^{C \times H \times W}$, which is fed into a convolutional layer with a $1 \times 1$ convolution kernel to get feature maps of local regions $A \in \mathbb{R}^{C \times H \times W}$. This process can be represented as:

$$
A=\operatorname{Sig}(\operatorname{Conv}(G))=\operatorname{Sig}\left(\bigcup_{k=1}^{K} A_{k}\right)
$$

where $\operatorname{Sig}(\cdot)$ is a sigmoid function and $\operatorname{Conv}(\cdot)$ represents a convolution function, $K$ denotes the number of channels, i.e., the number of local regions. And $A_{k} \in \mathbb{R}^{H \times W}$ is a local region of object, such as the tail of horses, the head of birds, or the stamens of flowers.

To further enhance the consistency and robustness of local feature maps, the self-attention mechanism is leveraged to model the relationship between different parts of the object. We first reshape the local feature map to $B \in \mathbb{R}^{K \times N}$, where $N=H \times W$. The modeling process can be represented as:

$$
P=R\left(\operatorname{Nor}(B) B^{T} B\right)
$$

where $R(\cdot)$ is reshape operation and $\operatorname{Nor}(\cdot)$ indicates the softmax normalization on the second dimension of a matrix. $\operatorname{Nor}(B) B^{T}$ can be regarded as attention maps, which models the 
relationship of different channels (i.e., different parts of object). Specifically, $B_{i}$ denotes feature map of $i$-th channel and $\operatorname{Nor}(B) B_{i, j}^{T}$ represents the spatial relationship between $i$-th channel and $j$-th channel. Then matrix multiplication is implemented between $\operatorname{Nor}(B) B^{T}$ and $B$, the result is reshaped to $P \in \mathbb{R}^{K \times H \times W}$, to strengthen the consistency of feature maps.

Compared with feature maps $A$, feature maps $P$ has better visual feature representation capacity as the semantic dependencies between different parts of the object is established. Moreover, local features $P$ can capture more detail information of objects than global features, resulting in better recognition capability on similar objects.

Global and local features contain complementary information of different scales. Global features have more global information of objects, while local features comprise more detail information of objects. Therefore, jointly learning global and local features can be used to obtain better visual representations, which leads to better ZSL performance. We fuse global feature map $G$ and local feature map $P$ to capture complementary information of global and local features. Specifically, element-wise multiplication is performed between global feature map $G$ and each local feature map $P_{k}$, and then we implement max-pooling operation on the result. Finally, $K$ feature vectors are concatenated together to get the visual representation $v\left(x_{f}\right)$ in visual space of fine-scale image $x_{f}$. The whole process is as follows:

$$
\boldsymbol{v}\left(x_{f}\right)=c\left(\bigcup_{k=1}^{K} g\left(P_{k} \otimes G\right)\right)
$$

where $\otimes$ indicates element-wise multiplication of tensors, $g(\cdot)$ and $c(\cdot)$ represent global maxpooling operation and concatenation operation, respectively. 


\subsection{The compatibility loss and prediction}

To associate the visual features with the semantic knowledge, many existing works adopt the bilinear compatibility score function parameterized by $\boldsymbol{W}$, which has the following form:

$$
F(x, y ; \boldsymbol{W})=\boldsymbol{\theta}(x) W \phi(y)
$$

where $\boldsymbol{\theta}(x)$ and $\phi(y)$ represent visual embedding of image $x$ and semantic embedding of class label $y$, respectively.

In our method, $\boldsymbol{v}(x)$ serve as the visual embedding of input image $x$, and then Eq. (8) can be reformulated as follows:

$$
F\left(x, y ; \boldsymbol{W}_{1}, \boldsymbol{W}_{2}\right)=\boldsymbol{v}(x) \boldsymbol{W}_{1} a^{y}
$$

where $\boldsymbol{W}_{\boldsymbol{l}}$ is the parameter that maps the visual embedding $v(x)$ to the semantic embedding $a^{y}$, and $\boldsymbol{W}_{2}$ represents the whole learnable parameters in the framework.

We choose $k \in \mathbb{R}^{d \times c}$ as semantic knowledge of $c$ classes with dimension $d$, and the class outputs of image $x$ on the final layer of $\mathrm{OCF}$ is:

$$
O\left(x ; \boldsymbol{W}_{1}, \boldsymbol{W}_{2}\right)=k^{T} \boldsymbol{W}_{1}^{T} \boldsymbol{v}(x)
$$

To learn the parameters $\left(\boldsymbol{W}_{1}, \boldsymbol{W}_{2}\right)$ of the model in an end-to-end manner, the loss function is as follows:

$$
\mathcal{L}=\frac{1}{N_{s}} \sum_{i=1}^{N_{s}} L\left(O\left(x_{i}^{s} ; \boldsymbol{W}_{1}, \boldsymbol{W}_{2}\right), y_{i}^{s}\right)
$$

where $N_{s}$ is the training sample size, given input image $x_{i}^{s}, L$ denotes the classification loss, which can be softmax loss, the mean square loss and cross entropy. And the weights of the cross-entropy loss layer used in our model are frozen as $k$ and fixed without updating during the training stage. 
In the proposed model, the tested unseen images can be directly mapped to semantic space that consists of user-defined attributes or visual descriptions, so the prediction of unseen images can be implemented in the semantic space. Specifically, given a tested image $x^{u}$, we map it to the semantic space to obtain semantic representation $\phi\left(x^{u}\right)=\boldsymbol{W}_{1}^{T} \boldsymbol{v}\left(x^{u}\right)$, and finally the location of the maximum compatibility score is chosen as the label of $x^{u}$ :

$$
y^{u^{*}}=\underset{c \in \mathcal{Y}^{u}}{\arg \max } \phi\left(x^{u}\right) a_{c}^{u}
$$

\section{Experiment}

\subsection{Datasets and experiment settings}

We test the effectiveness of our proposed model on three widely used datasets in ZSL: Animals with Attributes2 (AwA2) [5], Caltech-UCSD Birds 200-2011(CUB) [9] and Oxford Flowers (FLO) [8]. The example images of these three datasets are presented in Fig. 5, Fig. 6, and Fig. 7, respectively. Specifically, AwA2 is a coarse-grained dataset, which consists of 37322 images, 50 common animal categories, and 85 user-defined attributes. The fine-grained bird dataset CUB includes 11788 images from 200 categories, and 312-dim attribute vectors are utilized as semantic knowledge. FLO is a fine-grained dataset that includes 8189 images from 102 different types of flowers without attribute annotation. Then the visual description of FLO collected by [45] is utilized as the substitution of attributes in our experiments. Additionally, for AwA2 and CUB, we also evaluated our method on three other semantic knowledge, i.e., word2vec, glove, and hierarchies. In terms of size, word2vec and glove are 400 dimensional whereas hierarchies is 200 dimensional. More details about the datasets used in our experiment are listed in Table 1. 
Table 1 Summary of three ZSL datasets.

\begin{tabular}{ccccc}
\hline Dataset & Instances & Seen/Unseen & Detail & Semantic/dim \\
\hline CUB & 11788 & $150 / 50$ & Fine-grain & A/312 \\
AwA2 & 37322 & $40 / 10$ & Coarse-grain & A/85 \\
FLO & 8189 & $82 / 20$ & Fine-grain & V/1024 \\
\hline
\end{tabular}

A means attributes, and $\mathrm{V}$ means visual description vector

For a fair comparison with baseline, we adopt two dataset splits (as illustrated in [5]) for AwA2 and CUB, i.e., standard splits (SS), and the proposed splits (PS). For dataset FLO, we utilize the standard splits provided by [45]. And average class accuracy (ACA) is used to evaluate the quantitative performance of our proposed method.

\subsection{Implementation details}

Our proposed method is implemented in Pytorch. Pre-trained BASNet serves as the salient object detector to localize object region and pre-trained Resnet101 serves as the CNN backbone to extract visual features. For BASNet, the size of input images is same as the size of images in original dataset. And the size of the cropped and zoomed images is $224 \times 224$, which is the size of the $\mathrm{CNN}$ backbone input. At the same time, the output feature size of the CNN backbone is $2048 \times 7 \times 7$. SGD optimizer is adopted to optimize the objective function. Besides, momentum, and weight decay are set to 0.9 , and $5 \times 10^{-4}$, respectively, and the initial learning rate is selected from [0.001,0.003]. To train our model stably, the number of local regions $K$ is selected from $\left\{K \in \mathrm{N}_{+} \mid 10 \leq K \leq 50\right\}$. 


\subsection{Zero-shot classification results}

Our proposed method is compared with two groups of state-of-the-art methods: inductive and ResNet101 based methods, inductive end-to-end trainable CNN based methods. The first group consists of Direct Attribute Prediction (DAP) [19], Latent Embedding (LATEM) [22], Label Embedding (ALE) [20], Deep Visual-Semantic Embedding (DeViSE) [21], Structured Joint Embedding (SJE) [41], Embarrassingly Simple Zero Shot Learning (ESZSL) [42], and Semantic Auto Encoder (SAE) [17]. And Deep Embedding Model (DEM) [23], Latent Discriminative Features (LDF) [26], Coupled Dictionary Learning (CDL) [48], and SemanticGuided Multi-Attention model (SGMA) [40] are included in the second group. Average class accuracy (ACA) is adopted as an evaluation approach, which can be described as follows:

$$
a c c_{\mathcal{U}}=\frac{1}{\|\mathcal{U}\|} \sum_{c=1}^{\|\mathcal{U}\|} \frac{\# \text { correct predictions in } c}{\text { \#samples in } c}
$$

where $\|\mathcal{U}\|$ is the number of the test classes $\mathcal{U}$.

The ZSL experiment result is summarized in Table 2. It can be seen that our proposed method OCF achieves much better performance than all the compared approaches. And the accuracies on three datasets are $87.6 \%, 69.6 \%, 72.6 \%, 73.8 \%$, and $68.5 \%$ respectively, which are better than other compared methods.

\subsubsection{ZSL results on AwA2 dataset}

The zero-shot classification results of the proposed method OCF on AwA2 dataset are presented in the confusion matrix in Fig. 8. In the confusion matrices, the rows correspond to the prediction and the columns correspond to the ground truth.

As shown in Table 2, under SS setting, the impressive result $87.6 \%$ is obtained, which is 
4.2\% higher than the best result of LDF. Under PS setting, the performance of our proposed method is $1.7 \%$ higher than the best result of CDL (69.6\% vs. 67.9\%). The reason may be that OCF makes full use of complementary visual information of the global and local features of the input images. What's more, neglecting useless backgrounds information and paying attention to the discriminative regions further contribute to better ZSL performance.

Additionally, OCF outperforms the ResNet101based methods by a wide margin, such as DAP, ESZSL and SAE. And the performance of end-to-end trainable CNN based methods is usually better than the ResNet101based methods, which shows that end-to-end model leads to better ZSL performance.

Table 2 ZSL results (ACA, in \%) on AwA2, CUB and FLO.

The best results are marked in red and the second best are marked in blue.

\begin{tabular}{|c|c|c|c|c|c|c|}
\hline & \multirow{2}{*}{ Method } & \multicolumn{2}{|c|}{ AwA2 } & \multicolumn{2}{|c|}{ CUB } & \multirow{2}{*}{ FLO } \\
\hline & & SS & PS & SS & PS & \\
\hline \multirow{8}{*}{$\dagger$} & DAP [19] & 58.7 & 46.1 & 37.5 & 40.0 & - \\
\hline & LATEM [22] & 68.7 & 55.8 & 49.4 & 49.3 & 40.4 \\
\hline & ALE [20] & 80.3 & 62.5 & 53.2 & 54.9 & 48.5 \\
\hline & DEVISE & 68.6 & 59.7 & 53.2 & 52.0 & 45.9 \\
\hline & SJE [41] & 69.5 & 61.9 & 55.3 & 53.9 & 53.4 \\
\hline & ESZSL [42] & 75.6 & 58.6 & 55.1 & 53.9 & 51.0 \\
\hline & SAE [17] & 80.7 & 54.1 & 33.4 & 33.3 & 45.6 \\
\hline & TCN [49] & 71.2 & - & 59.5 & - & - \\
\hline \multirow{5}{*}{ 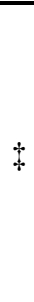 } & DEM [23] & - & 67.1 & 51.8 & 51.7 & 41.6 \\
\hline & LDF [26] & 83.4 & - & 67.1 & 67.5 & \\
\hline & CDL [48] & 79.5 & 67.9 & 54.5 & 54.5 & - \\
\hline & SGMA [40] & - & - & 70.5 & 71 & 65.9 \\
\hline & OCF(Ours) & 87.6 & 69.6 & 72.6 & 73.8 & 68.5 \\
\hline
\end{tabular}




\subsubsection{ZSL results on CUB dataset}

The confusion matric on CUB dataset under PS setting is shown in Fig. 9, from which we can see that OCF achieves higher precision on more challenging fine-grained dataset CUB. From Table 2, we can find that our result on CUB dataset is further improved to $72.6 \%$ and 73.8\% (SS and PS setting, respectively), which are $2.1 \%$ and $2.8 \%$ higher than the best result of SGMA.

There are three main observations: (1) Table 2 shows that our method outperforms all the compared methods. The confusion matrices in Fig. 7 illustrate the excellent performance of the OCF method on fine-grained dataset CUB. This is attributed to the effectiveness of the proposed local discriminative attention (LDA) module, which can discover subtle visual differences of objects with similar appearance. (2) OCF and SGMA are better than other methods under both SS and PS setting. These results clearly demonstrate the contribution of learning global and local discriminative features. (3) We can see that SGMA yields a worse performance than our method. This proves that it is useful to learning better visual features from the object-centric region.

\subsubsection{ZSL results on FLO dataset}

The confusion matrices of our method on FLO dataset are shown in Fig. 10. From Tables 2, we can see that the results on the fine-grained dataset FLO is further improved by our method OCF. And this verifies that considering the complementary information of global and local features and capturing object-centric visual features are critical for zero-shot classification tasks. Moreover, experiment results in Table 2 indicates that OCF and SGMA method outperform 
other methods on FLO dataset. This validates that learning discriminative visual features are more important for fine-grained zero-shot classification. Compared with SGMA, our method obtains $2.6 \%$ improvement, which proves the effectiveness of our proposed method.

\subsubsection{ZSL results with different semantic knowledge}

Table 3 ZSL results (ACA, in \%) on CUB, AwA2 with four different semantic knowledge.

The best results are marked in red and the second best are marked in blue.

\begin{tabular}{c|cccc}
\hline \multirow{2}{*}{} & \multicolumn{2}{|c}{ CUB } & \multicolumn{2}{c}{ AwA2 } \\
& SS & PS & SS & PS \\
\hline attribute & $\mathbf{7 2 . 6}$ & $\mathbf{7 3 . 8}$ & $\mathbf{8 7 . 6}$ & $\mathbf{6 9 . 6}$ \\
word2vec & 40.1 & 34.8 & 67.6 & 41 \\
glove & 41 & 35.2 & 71.4 & 46.1 \\
hierarchies & 26.8 & 24.5 & 62.7 & 48.7 \\
\hline
\end{tabular}

Moreover, for CUB and AwA2, our model is evaluated with four semantic knowledge (attribute, word2vec, glove, and hierarchies), and the results are shown in Table 3. From the results, we can find that our model with attributes achieves the best results on CUB and AwA2 under both SS and PS settings. It is largely because attribute information contains more abstract semantic knowledge as well as beneficial visual information, which lead to better performance when transferring knowledge from seen classes to unseen classes. In addition, for CUB, our model with glove achieves the second-best results under both SS and PS settings, which are $41 \%$ and $35.2 \%$, respectively. For AwA2, with glove, our model achieves 71.4\% under the SS setting, and with hierarchies, our model achieves $48.7 \%$ under the PS setting. Compared with word2vec, the word vector glove presents better performance in ZSL, indicating that glove provides more transferring semantics than word2vec. Although word vector presentation (i. e. word2vec and glove) achieve inferior performance than attribute, it will be more popular in the future ZSL 
task because of its easy accessibility. Finally, our model with hierarchies is inferior on both CUB and AwA2, and hierarchies is expected to capture more semantics in the ZSL task.

\subsection{Ablation study}

In this section, we prove the effectiveness of the object-centric region refine module, the GDA module, and the LDA module, respectively. We set our baseline to the model without the object-centric region refine network, GDA, and LDA. Moreover, we define the baseline model with the object-centric region refine module as FBaseline, GL represents the model with the GDA and LDA module. Then we add GDA, LDA, and GL to FBaseline and analyze the effectiveness of each module. Our oblation study experiment is carried out on CUB, AwA2 and FLO, and the result is shown in Fig. 11.

\subsubsection{The effectiveness of object-centric region refine module}

We take the original images of three datasets as the input of the CNN backbone to get the baseline, which is $62.7 \%, 74.5 \%$, and $57.7 \%$ on CUB, AwA2, and FLO, respectively. Then we substitute the fine-scale images for original images as the input of the $\mathrm{CNN}$ backbone to get the FBaseline. Compared with baseline, the ACA of the model with the object-centric region refine module increases by $2.6 \%, 4.1 \%$, and $1.9 \%$ on CUB, AwA2, and FLO, respectively. And this is mainly due to the elimination of the interference information, and the extraction of the object regions by the object-centric region refine module, which further illustrates that the learning of object regions can improve ZSL performance.

Besides, we further implement an experiment on the CUB and AwA2 datasets to study the effect of different salient object detectors. Four typical salient object detectors, i. e., EGNet [50], 
BASNet [51], PAGE-Net [53], and SCRN [54], are utilized to cooperate with our proposed method OCF to complete the ZSL task. And the results are presented in Table 4. Under the SS setting of the CUB dataset, our method with EGNet achieves the best average class accuracy (ACA), i. e., $72.9 \%$, which is $0.3 \%$ higher than that of our model with BASNet. Under the PS setting, OCF with BASNet ranks first with the 73.8\% ACA, and the result of OCF with EGNet is $73.5 \%$. Therefore, we can find that the effectiveness of EGNet and BASNet on the CUB dataset are comparable. On the AwA2 dataset, OCF with SCRN achieves the best performance under both SS and PS settings, which are better than that of OCF with BASNet. And the performance of OCF with PAGE-Net is relatively inferior to our models with other three salient object detectors. Besides, we also find that the same salient objector exhibits different performance on different datasets, for example, OCF with SCRN achieves the best performance on the AwA2 dataset, whereas inferior result on the CUB dataset. All in all, the choice of salient object detector is very important for the performance of our model, and BASNet is most effective in our experiment.

Table 4 ZSL performance (ACA, in \%) with different salient object detectors in the objectcentric region refine module. The best results are marked in red and the second best are marked in blue.

\begin{tabular}{c|cccc}
\hline & \multicolumn{2}{|c}{ CUB } & \multicolumn{2}{c}{ AwA2 } \\
& SS & PS & SS & PS \\
\hline OCF with EGNet [50] & $\mathbf{7 2 . 9}$ & 73.5 & 87.3 & 69.3 \\
OCF with BASNet [51] & $\mathbf{7 2 . 6}$ & $\mathbf{7 3 . 8}$ & $\mathbf{8 7 . 6}$ & 69.6 \\
OCF with PAGE-Net [53] & 70.3 & 72.6 & 87.2 & $\mathbf{6 8 . 7}$ \\
OCF with SCRN [54] & 71.6 & 71.8 & $\mathbf{8 7 . 9}$ & $\mathbf{7 0 . 1}$ \\
\hline
\end{tabular}




\subsubsection{The effectiveness of GDA, LDA and their fused product}

We put the GDA module behind the CNN backbone based on the FBaseline to illustrate the effectiveness of the GDA module. The GDA module can capture long-range visual features, some of which are not captured by the CNN backbone, so the output visual features of the model with the GDA module are expected to be more expressive. Compared with the FBaseline, FBaseline+GDA achieves $1.7 \%$ and $1.9 \%$ improvement on fine-grain datasets CUB and FLO, respectively. For coarse-grain dataset AwA2, FBaseline+GDA obtains more obvious improvement (4.2\%). We can see from the results that the GDA module further promotes ZSL performance.

Additionally, we add the LDA module to FBaseline. However, we notice that the model with only local regions has poor performance, which is $4.3 \%, 9.7 \%$, and $8.1 \%$ less than FBaseline on CUB, AwA2, and FLO, respectively. And the results are also worse than baseline on all three datasets, especially on AwA2 (8.7\% decline). Compared with global features, these local regions only provide the local features of the object, although some of them are discriminative, they can cause some information loss, resulting in poor generalization on the test stage.

To fulfill the advantages of global and local features, we add both GDA and LDA to FBaseline, and the model can be presented as FBaseline+GL. On AwA2, FBaseline+GL reaches 87.6\%, with an impressive gain over baseline and FBaseline (13.1\% and 9.2\%, respectively). And on FLO, compared with baseline and FBaseline, $10.8 \%$ and $8.9 \%$ improvement are obtained, respectively. For more challenging dataset CUB, FBaseline+GL achieves $73.8 \%$, 
which is $11.1 \%$ and $8.5 \%$ higher than baseline and FBaseline, respectively. The experiment results show that the combination of the GDA and LDA module contributes to ZSL performance. And our experiment further illustrates the effectiveness of learning object-centric complementary features.

\subsubsection{Influence of local feature dimension}

We utilize a Convolution layer with a $1 \times 1$ kernel to compress the feature channels in the LDA module, where the number of channels $K$ is a hyper-parameter that can be tuned. Let $K$ varies in $\{1,10,20,50,80,100\}$, and we observe its impacts on ZSL performance on dataset CUB and FLO, the result is shown in Fig. 12. And we observe to case: 1) the model using the LDA module to capture local features (LDA, marked in red), and 2) the model using the convolution layer with a $1 \times 1$ kernel to capture local features $(1 \times 1$ Conv, marked in green).

On CUB (PS setting), when $K=20$, LDA, and $1 \times 1$ Conv achieve the best results which are $73.8 \%$ and $71.9 \%$, respectively. For FLO, when $K=20$, LDA and $1 \times 1$ Conv reach the peaks at $68.5 \%$ and $67 \%$, respectively. And it can be concluded that LDA is consistently better than $1 \times 1$ Conv.

Moreover, we can see that our model with a small $K$ (approximately $K=20$ ) achieves better performance in nearly all cases. And we also find that ZSL performance will decrease to a different degree when $K$ is too large or too small, which can be explained by a small number of local features lead to information loss, and a large number of local features lead to information redundancy. 


\subsection{Visualization}

Original images, salient images, and fine-scale images of datasets AwA2 and CUB are shown in Fig. 13. As shown in the original images, most regions of the image are occupied by complex backgrounds, which affect the extraction of discriminative visual features. We use the BASNet to obtain saliency maps, and the BASNet can effectively predict the object location. In the saliency maps, object region is reserved, and other complex backgrounds are eliminated. Compared with the original images, we can find that objects take up most regions in the finescale images, and the fine-scale images can better reflect the global information and local details of the objects. Finally, with the help of the object-centric fine-scale images, ZSL models tend to learn more discriminative visual features, which benefit ZSL performance.

To better understand the effectiveness of our proposed model OCF in capturing discriminative visual features, t-SNE is used to visualize the distribution of the unseen features on AwA2 under PS setting, and the results are shown in Fig. 14. Compared with the features extracted by the pre-trained ResNet101, the data distributions of different classes (marked in different colors) of OCF is more compact and less separated. Therefore, our OCF model achieves better ZSL performance.

In addition, the OCF model with PS split is used to visualize what the learned regions look like, and unseen images from AwA2 and CUB are considered. It's obvious that OCF focuses on the object regions, which lead to better ZSL performance.

\section{Conclusion}

In this paper, we have studied the importance of the object-centric complimentary features 
for ZSL. Inspired by this idea, we proposed a novel deep embedding model, which consists of the object-centric region refine network and the complementary features learning network. And our proposed model, OCF, can discover object regions and obtain fine-scale images automatically. Moreover, OCF can capture complementary information of global and local features with the guidance of semantic knowledge and compatibility loss. Extensive experiments prove that our proposed method outperforms the state-of-the-art methods. As future work, we plan to expand our method to improve GZSL performance.

\section{Acknowledgment}

This work is supported by the National Natural Science Foundation of China (51805078), the National Key Research and Development Program of China (2017YFB0304200). 


\section{References}

[1] P. Morgado, N. Vasconcelos, Semantically consistent regularization for zero-shot recognition, in: CVPR, 2017, pp. 2037-2046.

[2] P. Peng, Y. Tian, T. Xiang, Y. Wang, M. Pontil, T. Huang, Joint semantic and latent attribute modelling for cross-class transfer learning, IEEE Trans. Pattern Anal. Mach. Intell. 7 (40) (2018) 1625-1638.

[3] W. L. Hoo, C. S. Chan, Recognizing unknown objects with attributes relationship model, Expert Systems with Application,42(23) 9279-9283.

[4] X. Li, M. Fang, D. Feng, H. Li, J. Wu, Prototype adjustment for zero-shot classification, Signal Processing: Image Communication,74 (2019) 242-252.

[5] Y. Xian, C. H. Lampert, B. Schiele, Z. Akata, ZSL-A comprehensive evaluation of the good, the bad and the ugly, IEEE Trans. Pattern Anal. Mach. Intell. 9 (41) (2019) 22512265.

[6] J. Sharad, S. Suraj, K. Nitin, First steps toward CNN based source classification of document images shared over messaging app, Signal Processing: Image Communication, 78 (2019) 32-41.

[7] Y. L. Cacheux, H. L. Borgne, M. Crucianu, Modeling inter and intra-class relations in the triplet loss for zero-shot learning, in: ICCV, 2019, pp.10333-10342.

[8] M. Nilsback and A. Zisserman, Automated flower classification over a large number of classes, in: ICVGIP, 2008, pp. 722-729.

[9] C. Wah, S. Branson, P. Welinder, P. Perona, and S. Belongie, The Caltech-Ucsd Birds-2002011 Dataset, California Institute of Technology, 2011.

[10] G. Xie, L. Liu, X. Jin, F. Zhu, et.al, Attentive region embedding network for zero-shot learning, in: CVPR, 2019, pp. 9384-9393.

[11] S. Rahman, S. Khan, F. Porikli, A unified approach for conventional zero-shot, generalized zero-shot, and few-shot learning, IEEE Trans. Image. Process. 11 (27) (2018) 5652-5667.

[12] S. Mirzaei, H. Van, S. Khosravani, Hyperspectral image classification using non-negative tensor factorization and 3D convolutional neural networks, Signal Processing: Image Communication. 76 (2019) 178-185.

[13] Y. Yu, Z. Ji, J. Guo and Y. Pang, Transductive zero-shot learning with adaptive structural embedding, IEEE Trans. Neural. Netw. Learn. Syst. 9 (29) (2019) 4116-4127.

[14] L. Niu, J. Cai, A. Veeraraghavan, L. Zhang, Zero-shot learning via category-specific visual-semantic mapping and label refinement, IEEE Trans. Image. Process. 2 (28) (2019) 
965-979.

[15] Y. Long, L. Liu, F. Shen, L. Shao, X. Li, Zero-shot learning using synthesised unseen visual data with diffusion regularization, IEEE Trans. Pattern Anal. Mach. Intell. 10 (40) (2018) 2498-2512.

[16] Y. Fu, T. M. Hospedales, T. Xiang, S. Gong, Transductive multi-view Zero-shot learning, IEEE Trans. Pattern Anal. Mach. Intell. 11 (37) (2015) 2332-2345.

[17] E. Kodirov, T. Xiang, S. Gong, Semantic autoencoder for zero-shot learning, in: CVPR, 2017, pp. 4447-4456.

[18] Hu T, Qi H, See better before looking closer: weakly supervised data augmentation network for fine-grained visual classification, 2019 arXiv:1901.09891.

[19] C. H. Lampert, H. Nickisch, S. Harmeling, Learning to detect unseen object classes by between-class attribute transfer, in: CVPR, 2009, pp. 951-958.

[20] Z. Akata, F. Perronnin, Z. Harchaoui, C. Schmid, Label-embedding for attribute-based classification, in: CVPR, 2013, pp. 819-826.

[21] A. Frome, G. S. Corrado, J. Shlens, S. Bengio, J. Dean, T. Mikolov, et al., Devise: A deep visual-semantic embedding model, in: NIPS, 2013, pp. 2121-2129.

[22] Y. Xian, Z. Akata, G. Sharma, Q. Nguyen, M. Hein, B. Schiele, Latent embeddings for zero-shot classification, in: CVPR, 2016, pp. 69-77.

[23] L. Zhang, T. Xiang, S. Gong, Learning a Deep Embedding Model for Zero-shot learning, in: CVPR, 2017, pp. 3010-3019.

[24] S. Changpinyo, W. Chao, B. Gong and F. Sha, Synthesized Classifiers for Zero-shot learning, in: CVPR, 2016, pp. 5327-5336.

[25] V. K. Verm and P. Rai, A simple exponential family framework for Zero-shot learning, in ECML, 2017, pp.792-808.

[26] Y. Li, J. Zhang, J. Zhang, K. Huang, Discriminative learning of latent features for zeroshot recognition, in: CVPR, 2018, pp. 7463-7471.

[27] L. Chen, Y. Yang, J. Wang, W. Xu, A. L. Yuille, Attention to scale: scale-aware semantic image segmentation, in: CVPR, 2016, pp. 3640-3649.

[28] R. Zellers, Y. Bisk, A. Farhadi, Y. Choi, From recognition to cognition: visual commonsense reasoning, in: CVPR, 2019, pp. 6720-6731.

[29] G. Xie, X. Zhang, S. Yan, C. Liu, SDE: A Novel Selective, Discriminative and Equalizing Feature Representation for Visual Recognition, International Journal of Computer Vision, 2 (124) (2017) 145-168.

[30] Y. Yu, Z. Ji, Y. Fu, J. Guo, Y. Pang, Z. Zhang, Stacked semantic-guided attention model 
for fine-grained zero-shot learning, in: NIPS, 2018, pp. 5995-6004.

[31] B. Chen, W. Deng, Hybrid-attention based decoupled metric learning for zero-shot image retrieval, in: CVPR, 2019, pp.2750-2759.

[32] P. Wang, L. Liu, C. Shen, Z. Huang, et. al., Multi-attention network for one shot learning, in: CVPR, 2017, pp. 2721-2729.

[33] H. Jiang, R. Wang, S. Shan, X. Chen, Transferable contrastive network for generalized zero-shot learning, in: ICCV, 2019, pp. 9765-9774.

[34] G. Xie, X. Zhang, W. Yang, M. Xu, S. Yan, C. Liu, LG-CNN: From local parts to global discrimination for fine-grained recognition, Pattern Recognition, 71 (2017) 118-131.

[35] A. Farhadi, I. Endres, D. Hoiem, D. Forsyth, Describing objects by their attributes, in: CVPR, 2009, pp. 1778-1785.

[36] G. Xie, X. Zhang, X. Shu, S. Yan, C. Liu, Task-driven feature pooling for image classification, in: ICCV, 2015, pp. 1179-1187.

[37] J. Fu, H. Zheng, T. Mei, Look closer to see better: recurrent attention convolutional neural network for fine-grained image recognition, in: CVPR, 2017, pp. 4476-4484.

[38] J. Zhang, M. Marszałek, S. Lazebnik, C. Schmid, Local features and kernels for classification of texture and object categories: A comprehensive study, IJCV 73 (2) (2007) 213-238.

[39] X. Wang, R. Girshick, A. Gupta, K. He, Non-Local Neural Networks, in: CVPR, 2018, pp. 7794-7803.

[40] Y. Zhu, J. Xie, Z. Tang, X. Peng, A. Elgammal, Semantic-guided multi-attention localization for zero-shot learning, in: NIPS, 2019, pp. 5276-5286.

[41] Z. Akata, S. Reed, D. Walter, Honglak. Lee, B. Schiele, Evaluation of output embeddings for fine-grained image classification, in: CVPR, 2015, pp. 2927-2936.

[42] B. Romera-Paredes, P. H. Torr, An embarrassingly simple approach to zero-shot learning, in: ICML, 2015, pp. 2152-2161.

[43] Y. Liu, J. Guo, D. Cai, X. He, Attribute Attention for Semantic Disambiguation in ZeroShot Learning, in: ICCV, 2019, pp. 6698-6707.

[44] X. Xu, I. W. Tsang, C. Liu, Complementary Attributes: A New Clue to ZSL, IEEE Trans. Cybern. (2019) doi: 10.1109/TCYB.2019.2930744.

[45] S. Reed, Z. Akata, H. Lee, B. Schiele, Learning deep representations of fine-grained visual descriptions, in: CVPR, 2016, pp. 49-58.

[46] M. Norouzi, T. Mikolov, S. Bengio, Y. Singer, J. Shlens, A. Frome, G. S. Corrado, J. Dean, Zero-shot learning by convex combination of semantic embeddings, in: ICLR, 2014, pp.1- 
9.

[47] H. Jiang, R. Wang, S. Shan, Y. Yang, X. Chen, Learning discriminative latent attributes for zero-shot classification, in: ICCV, 2017, pp. 4233-4242.

[48] H. Jiang, R. Wang, S. Shan, and X. Chen, Learning class prototypes via structure alignment for zero-shot recognition, in: ECCV, September 2018.

[49] H. Jiang, R. Wang, S. Shan, X. Chen, Transferable contrastive network for generalized zero-shot learning, in: CVPR, 2019, pp. 9765-9774.

[50] J. Zhao, J. Liu, D. Fan, Y. Cao, J. Yang, M. Cheng, EGNet: edge guidance network for salient object detection, in: ICCV, 2019, pp. 8779-8788.

[51] X. Qin, Z. Zhang, C. Huang, M. Dehghan, BASNet: Boundary-aware salient object detection, in: CVPR, 2019, pp.7479-7489.

[52] G. Xie, X. Zhang, S. Yan, C. Liu, Hybrid CNN and dictionary-based models for scene recognition and domain adaption, IEEE Transactions on Circuits and Systems for Video Technology, 6 (27) (2017) 1263-1274.

[53] W. Wang, S. Zhao, J. Shen, C. Steven, Salient object detection with pyramid attention and salient edges, in: CVPR, 2019, pp.1448-1557.

[54] Z. Wu, L. Su, Q. Huang, Stacked cross refinement network for edge-aware salient object detection, in: ICCV, 2019, pp. 7264-7273. 


\section{Figure captions:}

Fig. 1. An example of the effectiveness of global and local features.

Fig. 2. The framework of the object-centric complementary features (OCF) learning model.

Fig. 3. The framework of global discriminative attention (GDA) module.

Fig. 4. An illustration of the local discriminative attention (LDA) module.

Fig. 5. The example images in AwA2 dataset.

Fig. 6. The example images in CUB dataset.

Fig. 7. The example images in FLO dataset.

Fig. 8. Confusion matrices of the test results on unseen classes on AwA2 dataset.

Fig. 9. Confusion matrices of the test results on unseen classes on CUB dataset under PS setting.

Fig. 10. Confusion matrices of the test results on unseen classes on FLO dataset.

Fig. 11. The performance of variants on ZSL with PS setting.

Fig. 12. The influence of different local features dimensions K on CUB and FLO.

Fig. 13. The examples of the original images, saliency maps and fine-scale images.

Fig. 14. t-SNE visualization of unseen images on AwA2 under PS setting.

Fig. 15. Visualization of the learned regions on unseen images of different datasets. 
Fig.1:

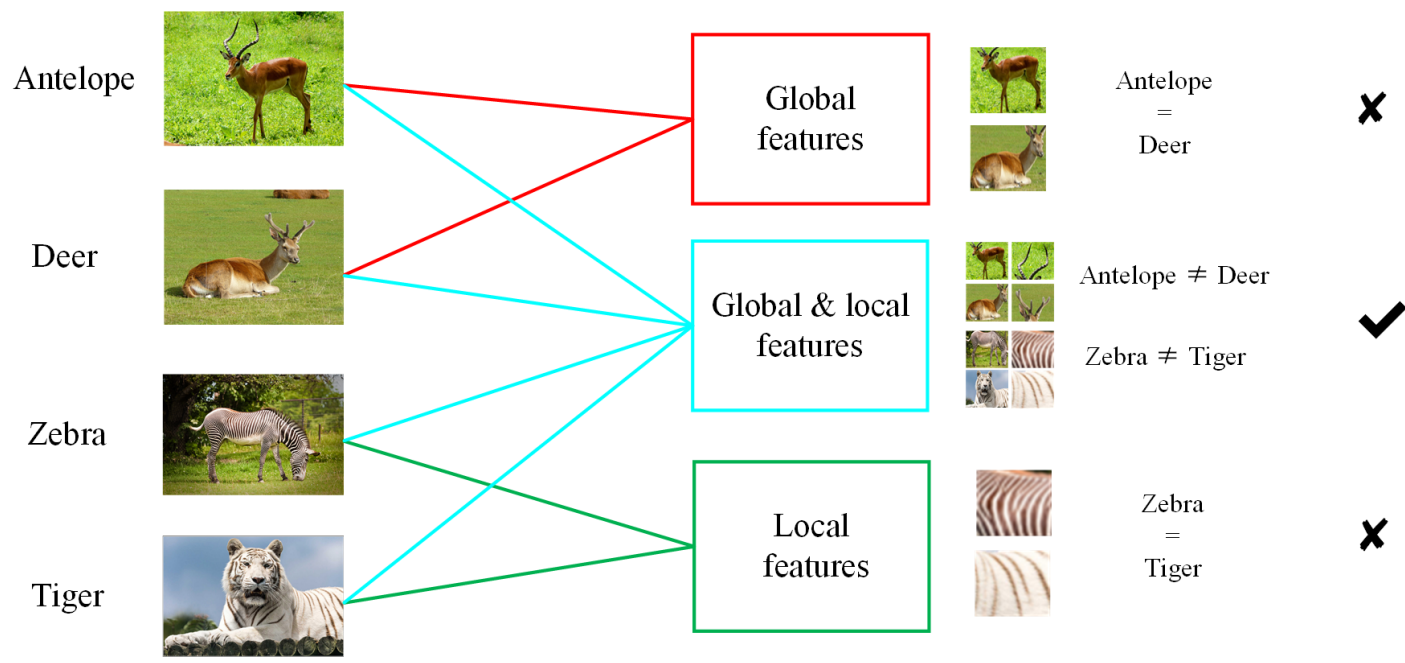

Fig. 1. An example of the effectiveness of global and local features. Antelope and deer are similar in their global features, only discriminative local features like the horn can distinguish them; Learning local features of zebra and tiger independently is difficult to classify them correctly, then global features are essential. 
Fig.2:

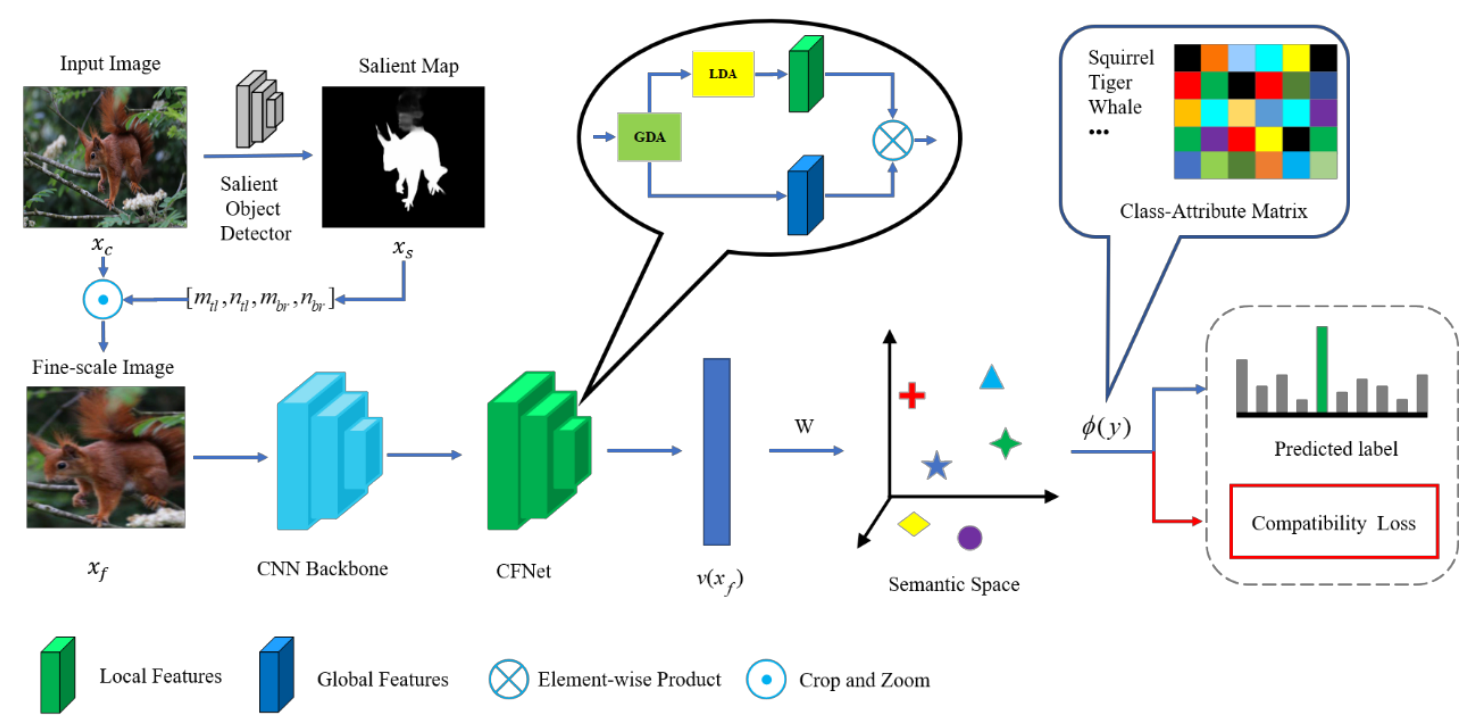

Fig. 2. The framework of the object-centric complementary features (OCF) learning model. The input image $x_{c}$ is fed into salient object detector to get salient map, and the object position $\left[m_{t l}, n_{t l}, m_{b r}, n_{b r}\right]$ is used to obtain fine-scale image $x_{f}$. Then $x_{f}$ are fed into CNN backbone and CFNet, in which GDA and LDA module are used to capture global and local features. And global and local features are fused to generate better visual features $\boldsymbol{v}\left(x_{f}\right)$. Finally, embedding to semantic space is leveraged, and compatibility loss is used for model training. 
Fig.3:

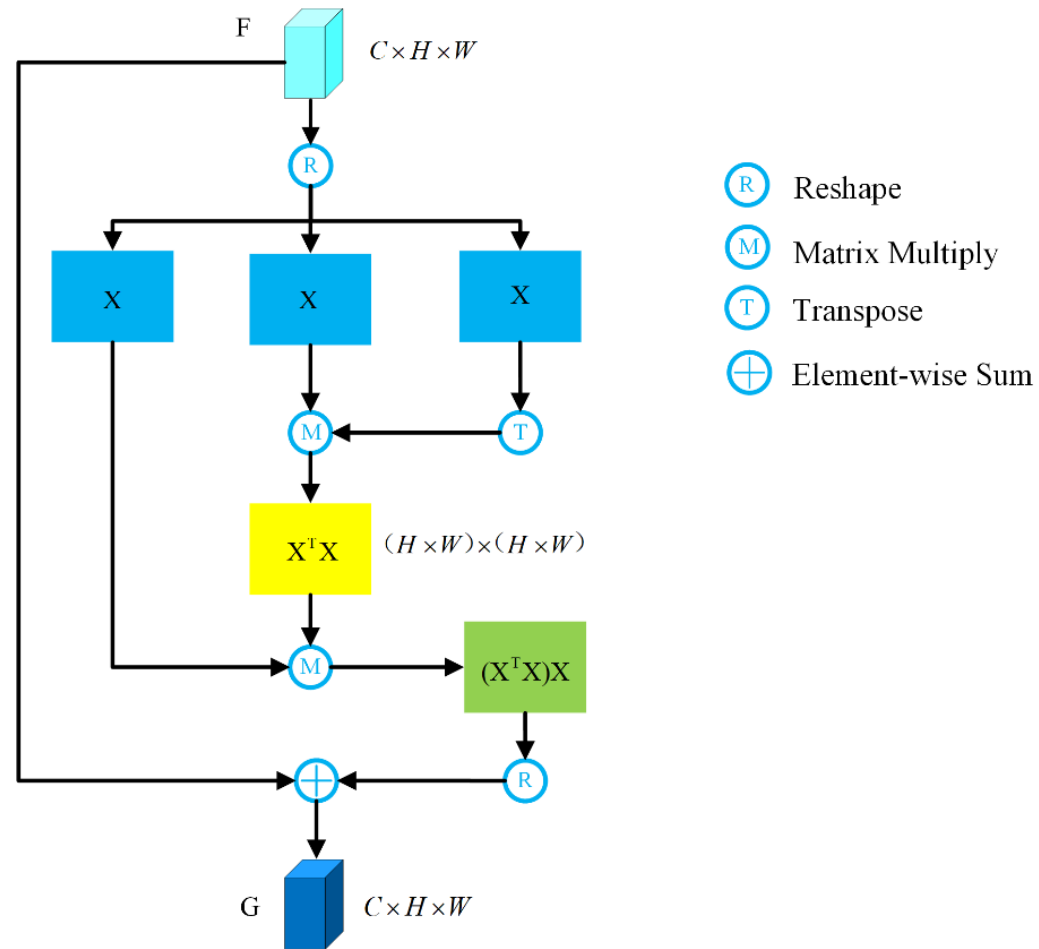

Fig. 3. The framework of global discriminative attention (GDA) module. 
Fig.4:

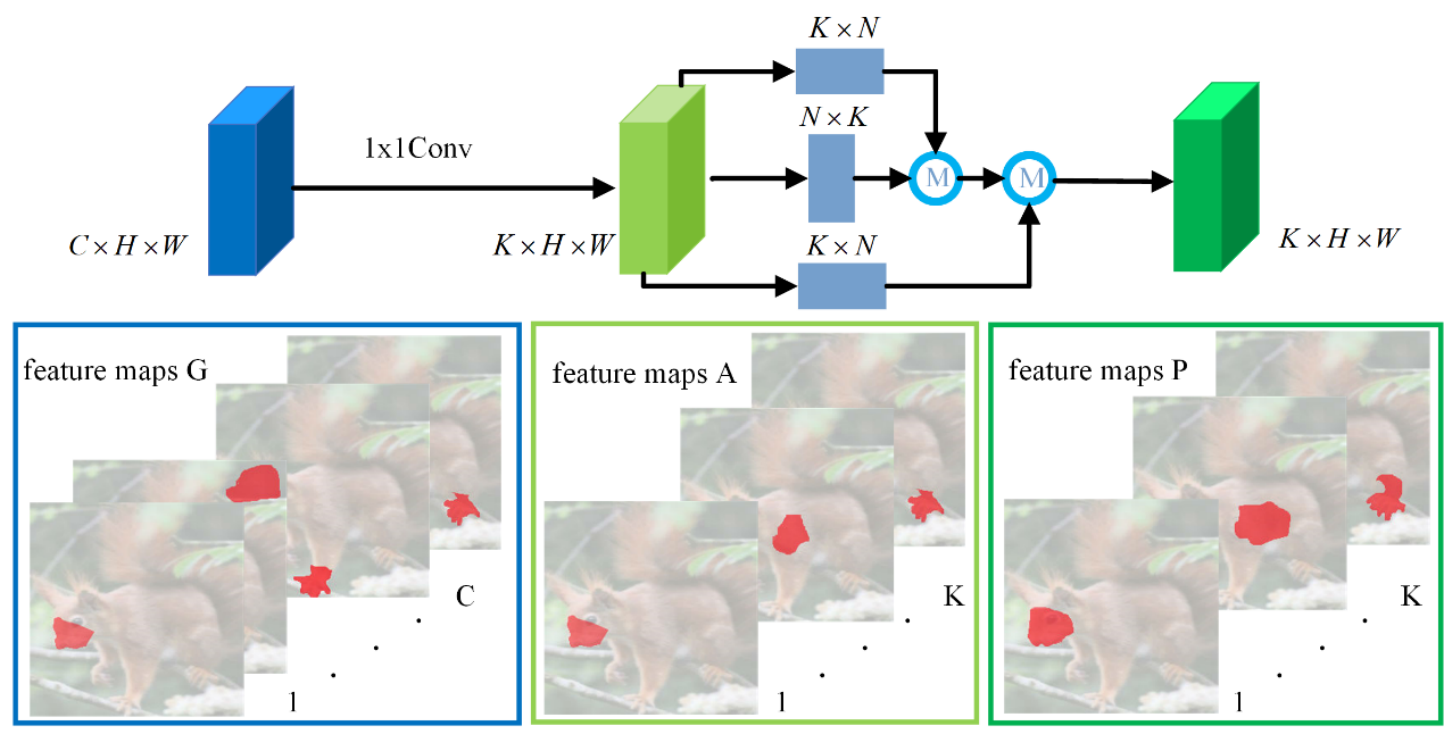

Fig. 4. An illustration of the local discriminative attention module (LDA). The global discriminative attention feature maps $G$ are put into a Convolution layer with a $1 \times 1$ kernel, then self-attention mechanism is used to capture more representative local features. M operation means matrix multiplication. 
Fig.5:

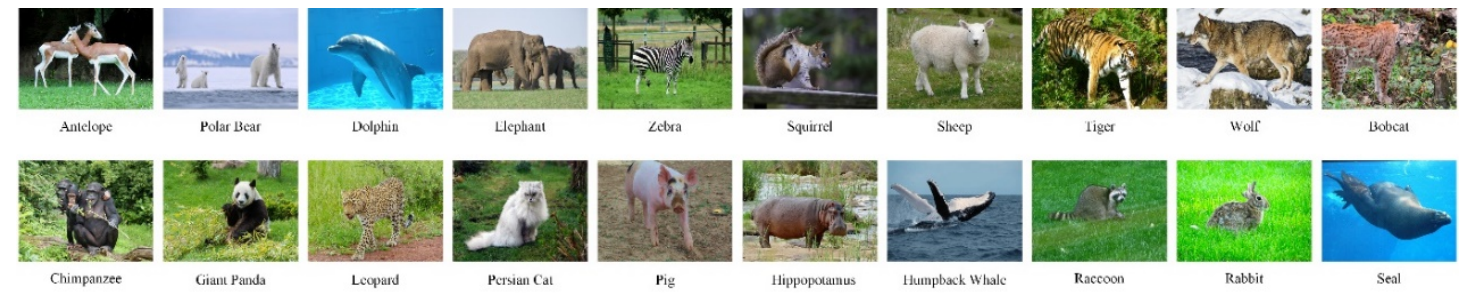

Fig. 5. The example images in AwA2 dataset. The top line is from the seen classes, and the bottom line is from the unseen classes. 
Fig.6:

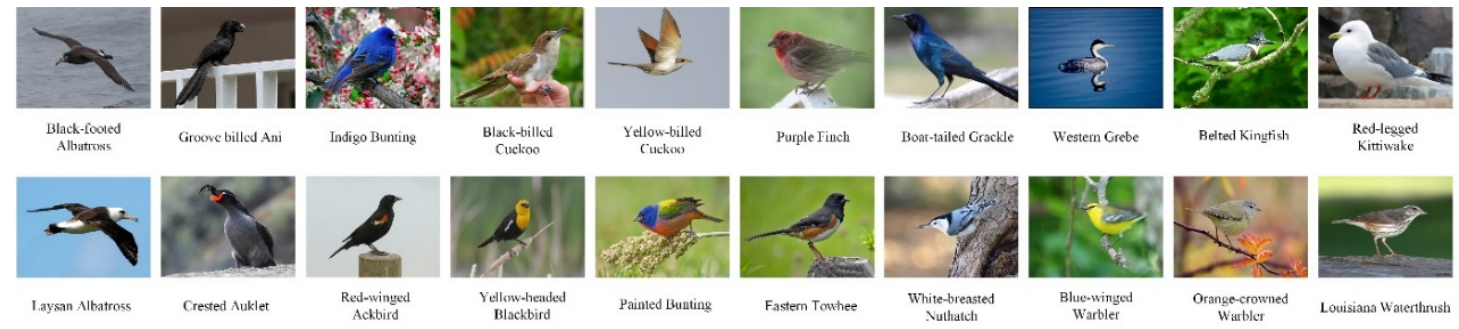

Fig. 6. The example images in CUB dataset. The top line is from the seen classes, and the bottom line is from the unseen classes. 
Fig.7:

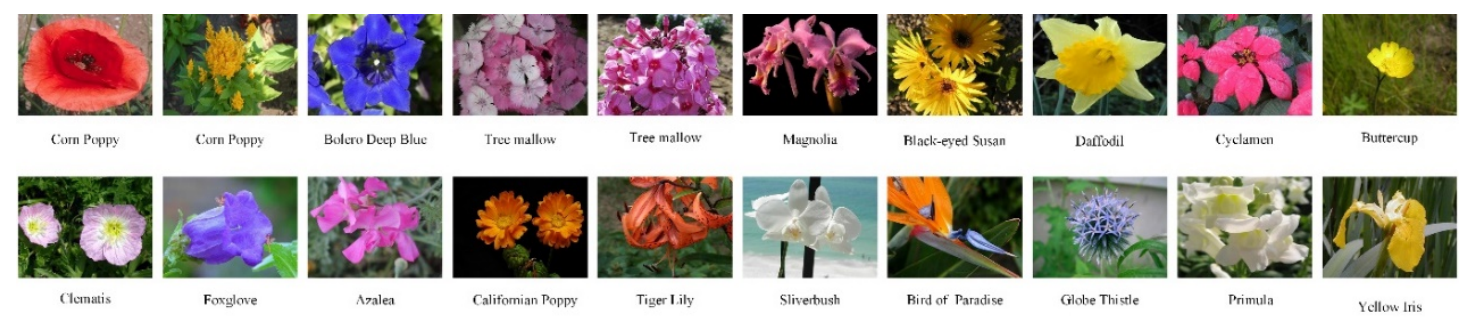

Fig. 7. The example images in FLO dataset. The top line is from the seen classes, and the bottom line is from the unseen classes. 
Fig.8:

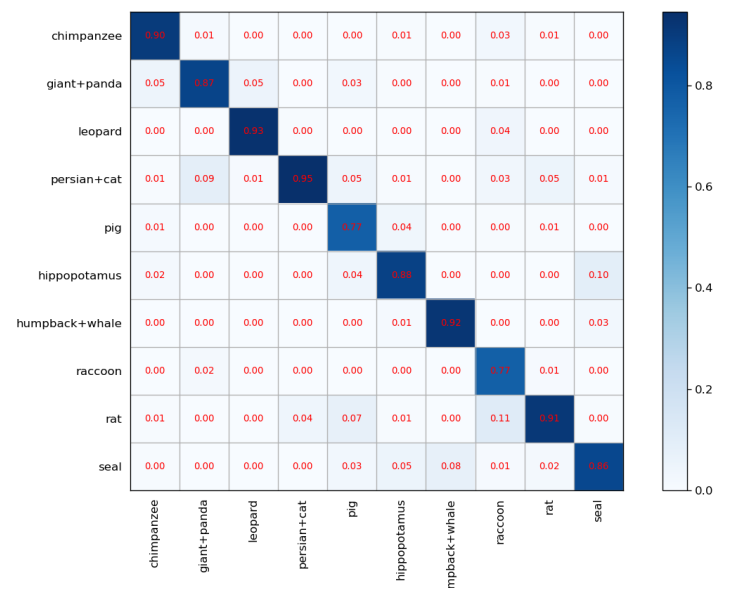

(a) SS setting

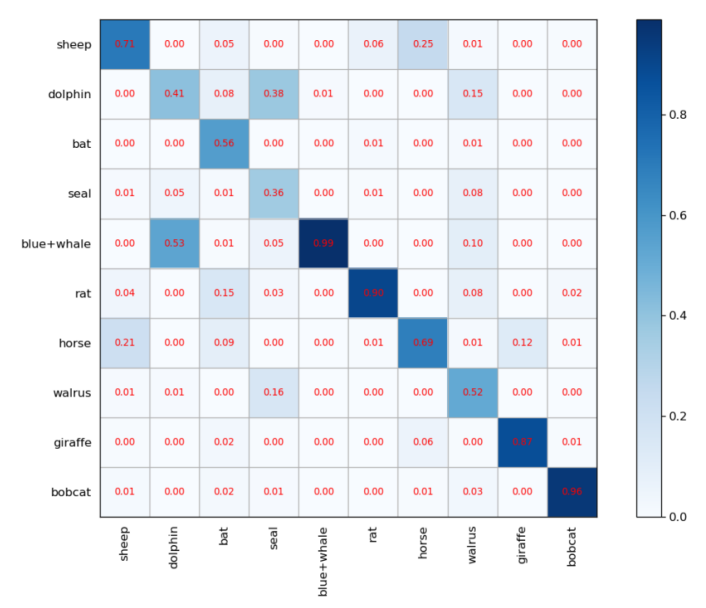

(b) PS setting

Fig. 8. Confusion matrices of the test results on unseen classes on AwA2 dataset (under both SS and PS setting). Diagonal numbers of indicate the correct prediction accuracy. the rows correspond to the prediction and the columns correspond to the ground truth. 
Fig.9:

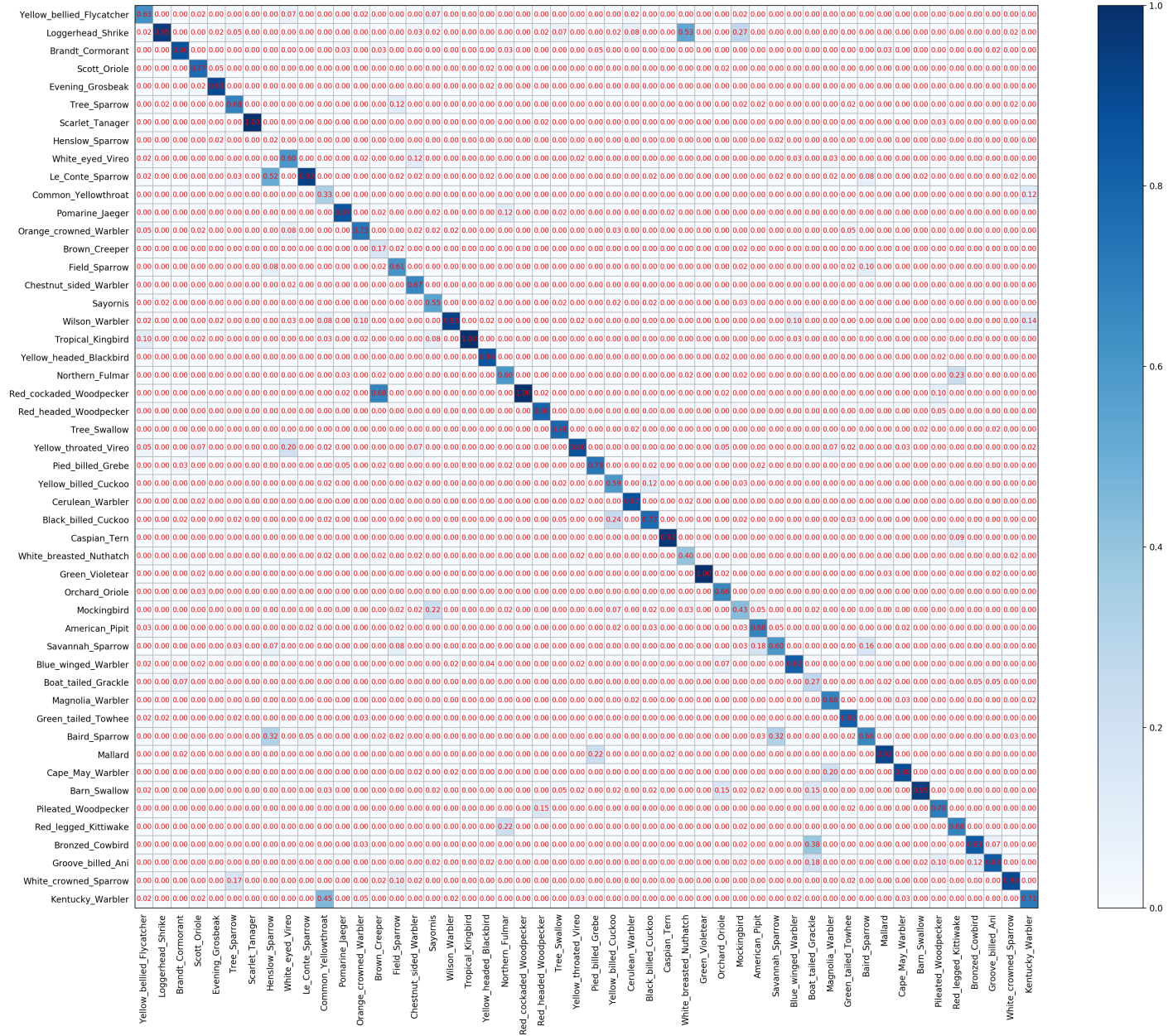

Fig. 9. Confusion matrices of the test results on unseen classes on CUB dataset under PS setting. Diagonal numbers of indicate the correct prediction accuracy. The rows correspond to the prediction and the columns correspond to the ground truth. 
Fig.10:

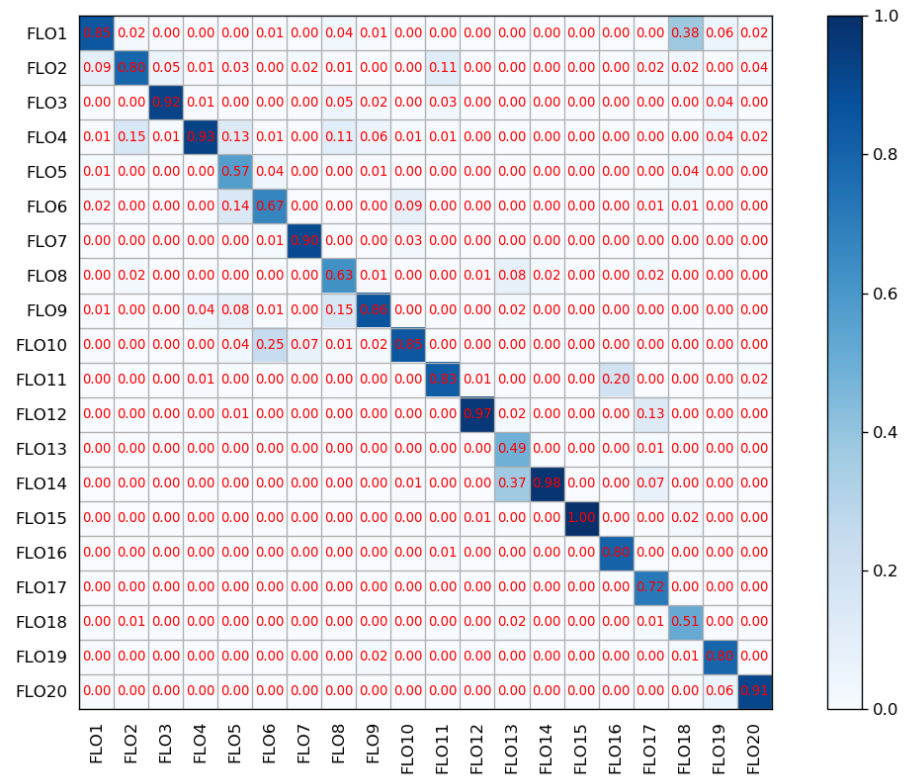

Fig. 10. Confusion matrices of the test results on unseen classes on FLO dataset. Diagonal numbers of indicate the correct prediction accuracy. The rows correspond to the prediction and the columns correspond to the ground truth. 
Fig.11:

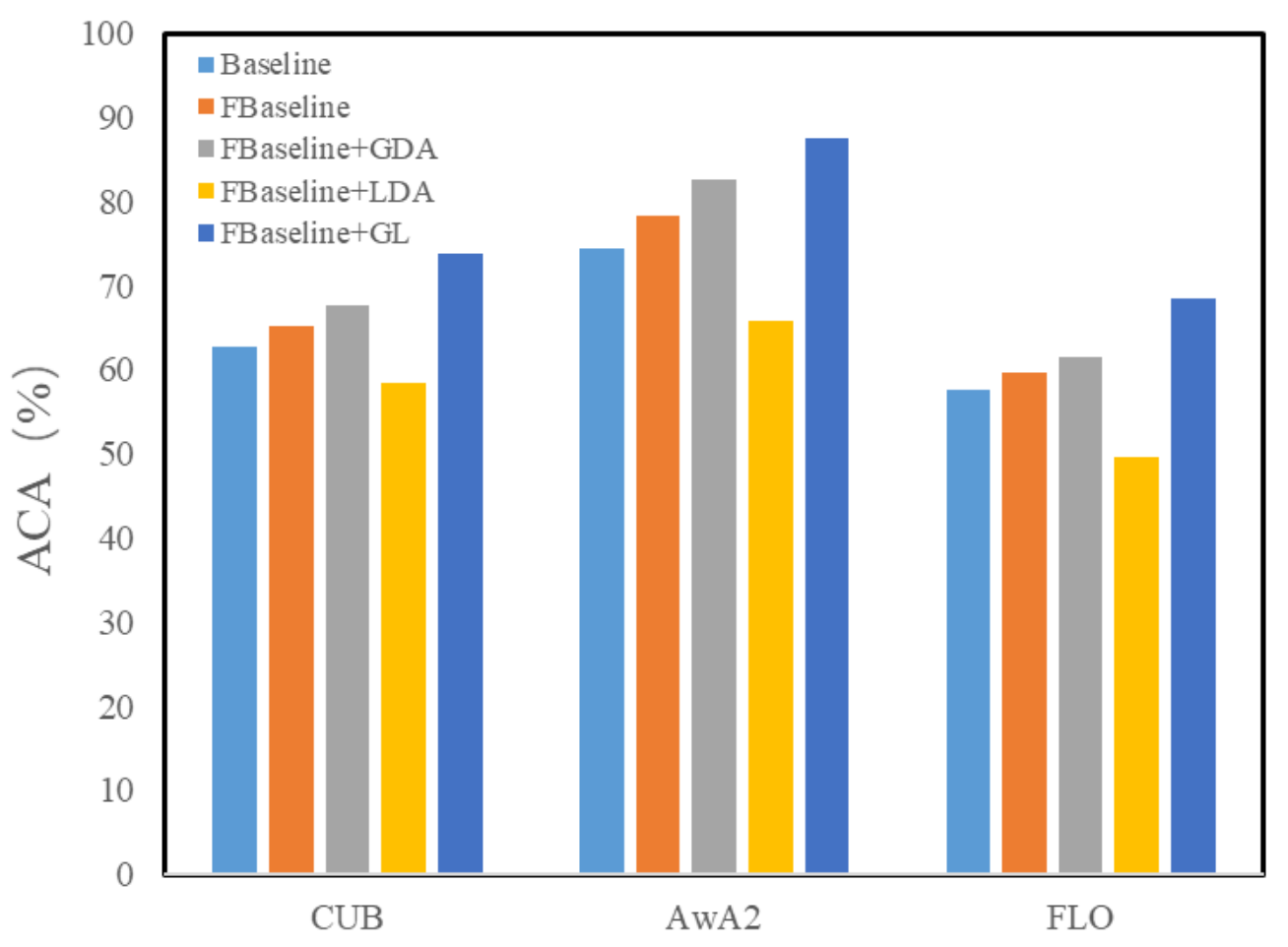

Fig. 11. The performance of variants on ZSL with PS setting. FBaseline means Baseline with fine-scale input images, while GDA, LDA, GL represent global features, local features, and their fused product, respectively. 
Fig.12:
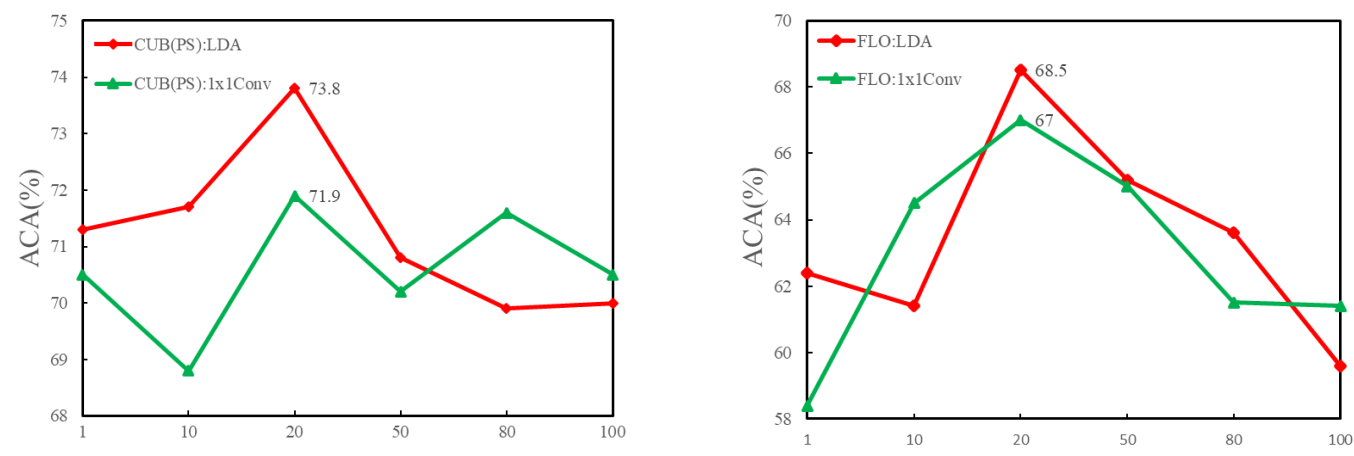

Fig. 12. The influence of different local features dimensions $\mathrm{K}$ on CUB and FLO, respectively. 
Fig.13:
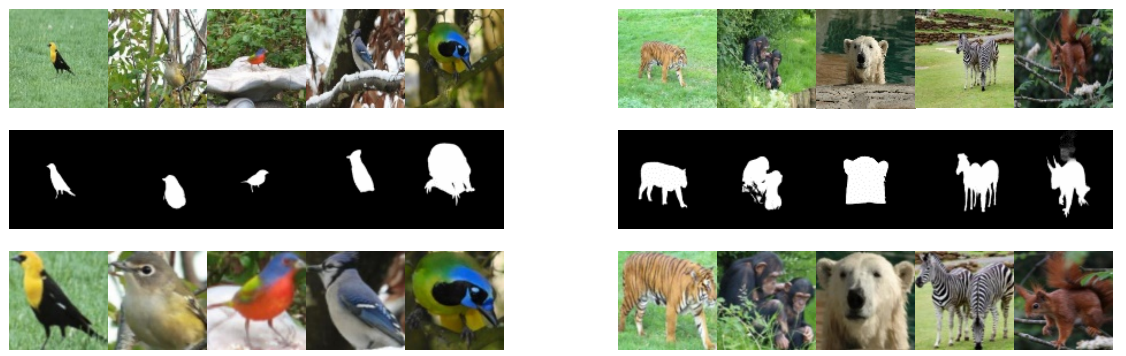

Fig. 13. The examples of the original images, saliency maps and fine-scale images. 
Fig.14:

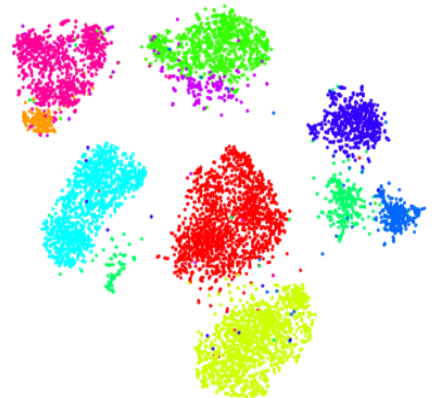

(a) Pre-trained ResNet101

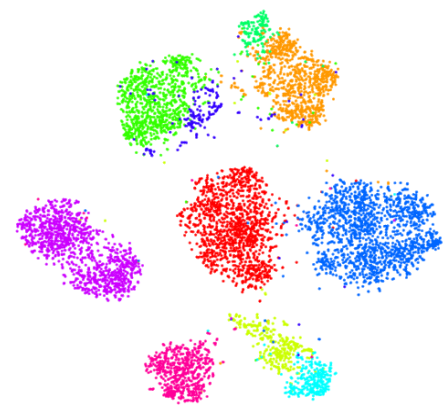

(b) Our proposed OCF

Fig. 14. t-SNE visualization of unseen images on AwA2 under PS setting. 
Fig.15:

AwA2

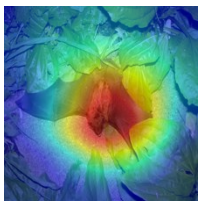

Bat

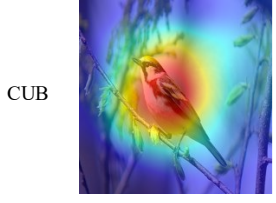

Chestnut-Sided

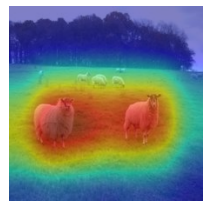

Sheep

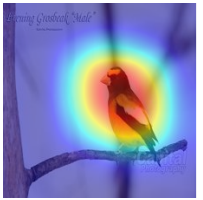

Evening Grosbeak

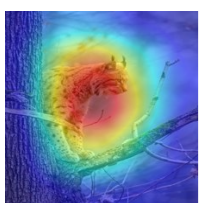

Bobcat

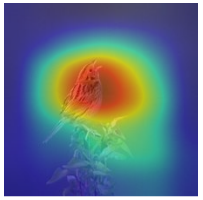

Henslow Sparrow

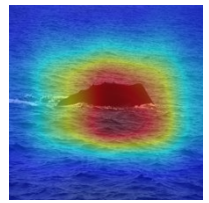

Blue+whale

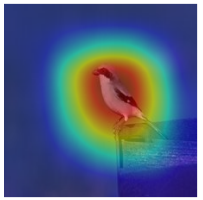

Loggerhead Shrike

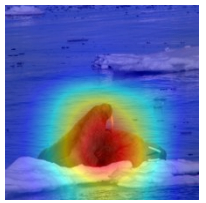

Walrus

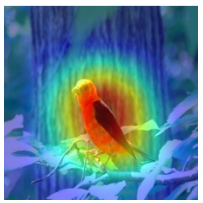

Scarlet Tanager

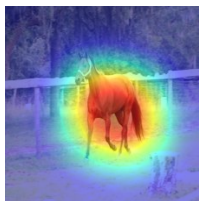

Horse

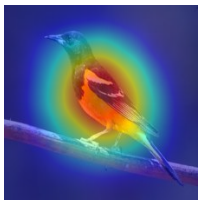

Scott Oriole

Fig. 15. Visualization of the learned regions on unseen images of different datasets. Lighter color indicates higher score. 